\title{
Cell-based in vitro systems for continuous monitoring of bioenergetics and cell impedance
}

\author{
Ehret R., Drechsler S., Kob A., Wego M. and Ortinau S. \\ Bionas $\mathrm{GmbH}$ \\ Friedrich-Barnewitz-Strasse 3 \\ 18119 Rostock
}

\section{Introduction}

The identification of pharmacodynamic properties of drug substances and the prediction of toxicity in early phases of drug development are major challenges of advanced in vitro methods. Therefore the evaluation of short-term effects of receptor signaling studies and the investigation of long-term effects mediated by toxins and xenobiotics are essential. In addition, drug interactions with target cells are of major interest. The Bionas Discovery label-free assay family measures physiological functions of cell lines and primary cells using non-invasive assays in a physiological controlled environment which provides information about pharmacological and toxicological as well as metabolic properties of the cells. The systems monitor cellular changes, including cytotoxic effects, by generating bioenergetic (glycolysis and mitochondrial respiration) and cellular impedance profiles as well as the regeneration potential of cells. Data may either be generated in long-term studies over hours to days or via short-term detection of cellular responses as required in toxicology, oncology and drug discovery.

Glycolysis and mitochondrial respiration are two major cellular processes for the generation of ATP. The activation of energy consuming reactions, such as signal transduction lead to a raised consumption of oxygen. This in turn leads to an increased release of acidic breakdown products and an increasing oxygen deficit in the medium. An additional factor which describes properties of the cell is the impedance/adhesion. The measurement of impedance/adhesion is used to detect the presence of electrically insulating cell membranes near the measurement electrodes [1,2]. The results are influenced by several parameters like distance from the membrane to the electrodes, electrode surface covered by the membranes, membrane integrity, cell-cell contacts and finally cell shape and morphology.

\section{Materials and Methods APAP toxicity assay}

Primary human and rat hepatocytes were seeded onto collagen precoated Bionas ${ }^{\circledR}$ metabolic chips SC1000 at a density of 150000 cells per chip in chemically defined Human Hepatocyte Maintenance Medium (HHMM) or Hepatocyte Growth Medium (HGM) + 40 ng/ml HGF +20 ng/ml EGF for the rat cells. HepG2 cells (human hepatocellular liver carcinoma cell line) were seeded on Poly-L-Lysin coated chips at a density of 200000 cells in DMEM with FCS, glutamine and antibiotics. All cells were incubated for $24 \mathrm{~h}$ at $5 \% \mathrm{CO} 2$ and $37{ }^{\circ} \mathrm{C}$. For the measurement, the baseline of acidification, respiration and impedance were determined in a weakly buffered (1 mM HEPES) running medium (RM) for $4 \mathrm{~h}$, followed by a $24 \mathrm{~h}$ application of $5 \mathrm{mM}$ acetaminophen (APAP). After the removal of APAP from the medium, potential regeneration effects could be detected. The medium is fed over the cells and the media flow is stopped periodically, measuring the changes of $\mathrm{pH}$ and oxygen consumption [3-5]. In the pump phase the "used" medium is substituted with fresh medium. Typically the stop and pump phases last 2-4 minutes each.

\section{Paclitaxel toxicity assay}

The impedance technology provides cellular information about cell viability, morphology, adhesion/confluence, proliferation and membrane integrity. A549 cells (human lung carcinoma cell line) were plated in Bionas Discovery adcon plates at a density of 10000, 15000 of 20000 cells per well and monitored with the Bionas Discovery adcon reader. After monitoring cell adhesion and proliferation for $24 \mathrm{~h}$, paclitaxel was added to the cells at different concentrations (1 nM, $10 \mathrm{nM}$ and $100 \mathrm{nM}$, prediluted in growth medium) for $48 \mathrm{~h}$. At the bottom of each well interdigitated electrodes are integrated. In contrast to the Bionas Discovery 2500 system for APAP toxicity testing, no perfusion/pump cycles are necessary. The impedance is measured by applying an alternative voltage to the electrodes at a fixed frequency of $10 \mathrm{kHz}$. Values for the impedance for each sensor/well are recorded at specific time intervals [6]. 


\section{Results and Discussion}

\section{Long-term monitoring of APAP toxicity (bioenergetics and impedance)}

Online and long-term measurements provide valuable information about the metabolic processes of toxic compounds. Figure 1 shows the result of the toxicity testing with $5 \mathrm{mM}$ APAP in primary human and rat hepatocytes and the HepG2 cell line.

The glycolytic activity (Fig. 1A) of HepG2 cells and primary rat hepatocytes was inhibited by $20 \%$ and $40 \%$, respectively. Whereas regeneration was seen in rat hepatocytes, HepG2 cells did not show any regeneration effects after removing APAP from the medium. Acidification rates of human hepatocytes showed a slight increase of the acidification rates during APAP application compared to untreated cells. Measurement of oxygen consumption (Fig. 1B) in the presence of APAP indicated strongly reduced respiration rates in all three cell types with a complete regeneration of human, a partly regeneration of rat and almost no regeneration of HepG2 cells. In addition, the measured cellular impedance displayed unique profiles for each cell preparation. Impedance of human hepatocytes was hardly affected by APAP, whereas the impedance of rat hepatocytes decreased continuously during the time of APAP administration. The impedance of HepG2 cells slightly decreased with the onset of APAP application, but showed a continuous decrease after $16 \mathrm{~h}$

Generally, rat hepatocytes reacted more sensitive to APAP as the acidification rate and the cell impedance/adhesion were affected more strongly compared to human cells. In all parameters measured, rat hepatocytes did not show complete recovery to basal values, indicating an irreversible damage of the cells after the exposure to APAP. HepG2 cell reactions to APAP were similar to primary rat cells with a different course of the acidification rate and an earlier decrease of cell impedance/ adhesion. The temporal progression of cellular reactions caused by the application of APAP on primary human and rat hepatocytes as well as on the HepG2 cell line could be analyzed successfully. Respiration was inhibited by APAP in all three cell types, whereas cellular acidification rates and cell impedance/adhesion were affected by APAP only in rat hepatocytes and HepG2 cells. The comparison of the temporal analysis of the cellular reaction of different cell types to bioactive compounds provided important information on the pharmacodynamics of APAP and the individual responses of cells from several sources.
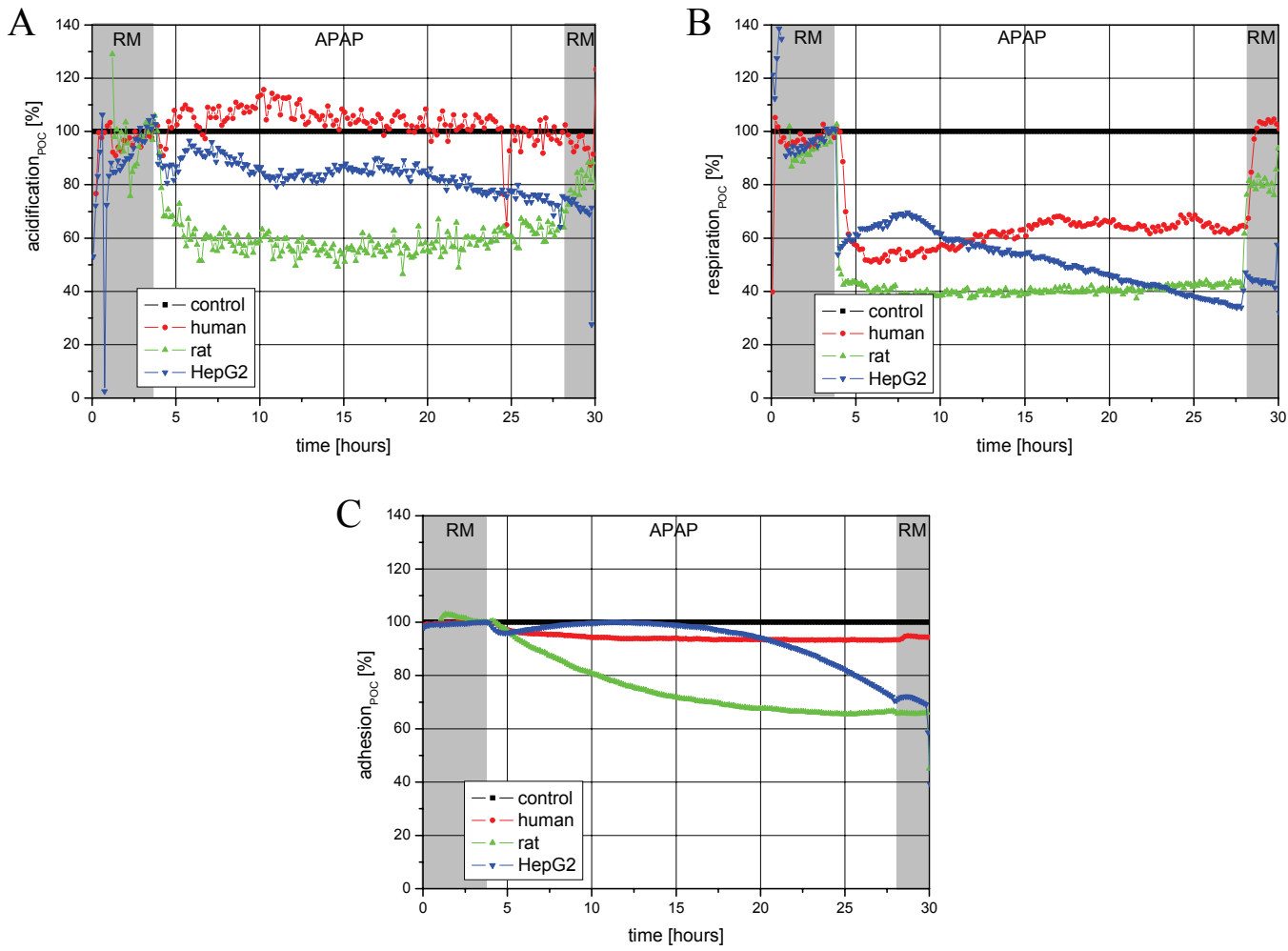

Figure 1: Acidification rates (A), respiration rates (B) and cell impedance/adhesion $(C)$ of primary human (red) and rat (green) hepatocytes and HepG2 cells (blue). After a prerunning phase of 4 h (grey area, RM) APAP was applied for $24 \mathrm{~h}$ (white area) followed by a regeneration phase (grey area, RM) without APAP. 
Dynamic monitoring of paclitaxel toxicity (impedance

The impedance technology offers the opportunity to online monitor the adhesion and growth of cells in combination with drug interactions. Figure 2 describes the monitoring of three different cell numbers of A549 cells from the time point of seeding (A: 10000 cells, B: 15000 cells and C: 25000 cells). After $24 \mathrm{~h}$ paclitaxel was added to the cells at three different concentrations (1 nM, $10 \mathrm{nM}$ and $100 \mathrm{nM})$ for $48 \mathrm{~h}$. Paclitaxel is a chemotherapeutic agent, which stabilizes microtubules of cells and therefore inhibits mitosis. Within the first $24 \mathrm{~h}$, adhesion, spreading and proliferation of A549 cells was monitored continuously. After that, 25000 cells did not show any further proliferation compared to 15000 and 10000 cells, which still showed further increase in impedance values.

The administration of paclitaxel strongly influenced cellular dynamics of all three different cell numbers. For the highest concentration, the impedance values immediately decreased in the same dynamic range for the individual cell numbers. A stop of proliferation or even cytotoxic effects have been initially visible. For $100 \mathrm{nM}$ of paclitaxel, the intensity of the effect is the same for all three different cell numbers. However, after $24 \mathrm{~h}$ of treatment, the effect of the highest concentration of paclitaxel is completely reversed and the cells recovered or even started to proliferate again, resulting in the same impedance values as in the beginning of the treatment at $24 \mathrm{~h}$.

From these results one can clearly assume, that the cell number is a major factor for the design of an experimental assay, as the number of plated cells contributes to the result. In addition, the data emphasize the importance and significance of a real-time data collection, as unexpected or reversible drug effects may occur.
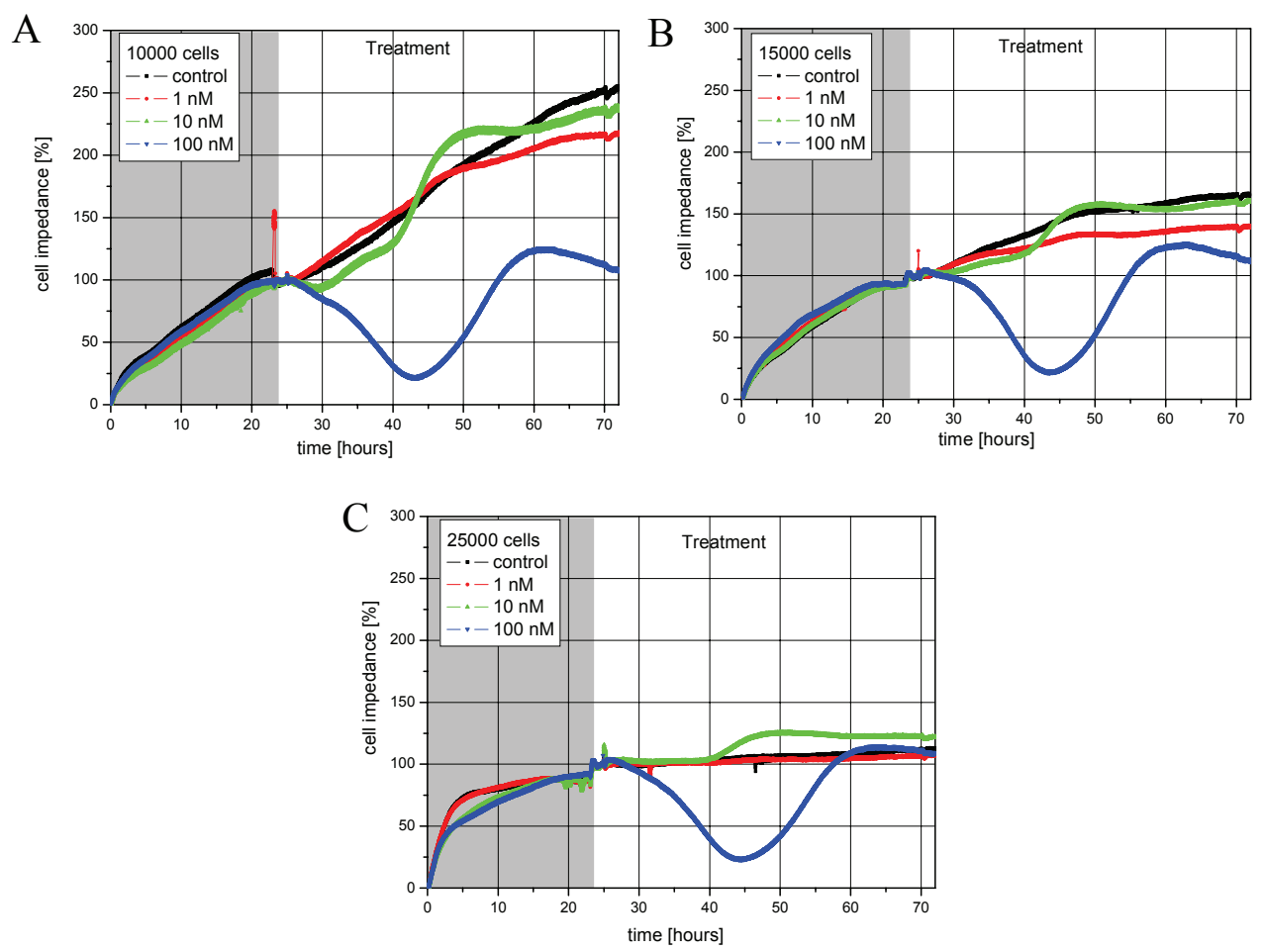

Figure 2: Dynamic monitoring of A549 cells and paclitaxel administration for $48 \mathrm{~h}$. After a $24 \mathrm{~h}$ proliferation phase (grey area), cells were treated with paclitaxel for $48 \mathrm{~h}$ at various concentrations (white area).

\section{Conclusions}

The Bionas Discovery label-free assay family allows a real-time monitoring of cellular processes. The multi-parameter technology is capable of analyzing extracellular acidification, respiration and cell impedance/adhesion in short and long-term studies, while the single-parameter device is a versatile instrument for impedance measurement in a higher throughput format. Compared to conventional end point assays the methods benefits from the measurement of transient effects and kinetic parameters.

The combination of these cellular parameters with the functionality of a temporal resolution of compound effects provides a valuable tool for cell-based functional studies gaining deeper insights into the dynamic of cell metabolism. 


\section{Literature}

[1] Ehret, R., Baumann, W., Brischwein, M., Schwinde, A., Stegbauer, K., Wolf, B.: Monitoring of cellular behaviour by impedance measurements on interdigitated electrode structures. Biosensors \& Bioelectronics, (1997):12(1):29-41

[2] Ehret, R., Baumann, W., Brischwein, M., Schwinde, A., Wolf, B.: On-line control of cellular adhesion with impedance measurements using interdigitate electrode structures. Medical \& Biological Engineering \& Computing, (1998) 36:365-370

[3] Thedinga, E., Kob, A., Holst, H., Keuer, A., Drechsler, S., Niendorf, R., Baumann, W., Freund, I., Lehmann, M., Ehret, R.: Online monitoring of cell metabolism for studying pharmacodynamics effects. Toxicology and Applied Pharmacology, (2007) 220: 33-44

[4] Thedinga E, Ullrich A, Drechsler S, Niendorf R, Kob A, Runge D, Keuer A, Fruend I, Lehmann M, Ehret R.:In vitro system for the prediction of hepatotoxic effects in primary hepatocytes. ALTEX, (2007) 24(1): 22-34

[5] Ceriotti L., Kob A., Drechsler S., Ponti J., Thedinga E., Colpo P., Ehret R., Rossi F.: Online monitoring of BALB/3T3 metabolism and adhesion with multiparametric chip-based system. Analytical Biochemistry, (2007) 371(1): 92-104

[6] Ceriotti L, Ponti J, Broggi, F, Kob A, Drechsler S, Thedinga E, Colpo P, Sabbioni, E, Ehret R, Rossi F.: Real-time assessment of cytotoxicity by impedance measurement on a 96-well plate. Sensors and Actuators B, (2007) 123(2): 769-778 